\title{
Correction to: Improvement of seedling and panicle blast resistance in Xian rice varieties following Pish introgression
}

\author{
Ning Xiao $($ ) Yunyu Wu • Zhiping Wang • Yuhong Li • Cunhong Pan • Xiaoxiang Zhang • \\ Ling Yu • Guangqing Liu • Changhai Zhou • Hongjuan Ji • Niansheng Huang • \\ Min Jiang $\cdot$ Zhengyuan Dai $\cdot$ Aihong Li
}

Published online: 31 May 2019

(C) Springer Nature B.V. 2019

\section{Correction to: Mol Breeding (2018) 38: 142 https://doi.org/10.1007/s11032-018-0899-6}

The original article unfortunately contains an incorrect grant number in the funding information.

The funding information with correct grant number should be:

This study was supported by the National Key R \& D Program of China [grant numbers 2017YFD0100304 and

The online version of the original article can be found at https://doi.org/10.1007/s11032-018-0899-6

N. Xiao $\cdot$ Y. Wu $\cdot$ Z. Wang $\cdot$ Y. Li $\cdot$ C. Pan $\cdot$ X. Zhang $\cdot$

L. Yu · G. Liu · C. Zhou · H. Ji • N. Huang • M. Jiang

Z. Dai $\cdot$ A. Li $(\bowtie)$

Institute of Agricultural Sciences for Lixiahe Region in Jiangsu,

Yangzhou 225009, China

e-mail: yzlah@126.com

N. Xiao $\cdot$ Y. Wu $\cdot$ Z. Wang $\cdot$ Y. Li $\cdot$ C. Pan $\cdot$ X. Zhang $\cdot$

L. Yu - G. Liu - C. Zhou - H. Ji • N. Huang - M. Jiang •

Z. Dai · A. Li

Jiangsu Collaborative Innovation Center for Modern Crop

Production, Nanjing 210095, China

N. Xiao $\cdot$ Y. Wu $\cdot$ Z. Wang $\cdot$ Y. Li $\cdot$ C. Pan $\cdot$ X. Zhang $\cdot$

L. Yu · G. Liu • C. Zhou - H. Ji • N. Huang · M. Jiang •

Z. Dai · A. Li

National Rice Industry Technology System of Yangzhou Comprehensive Experimental Station, Yangzhou, Jiangsu

Province, China
2017YFD0100402], the Project of Breeding of Key New Varieties in Jiangsu Province [grant number PZCZ201702], the Jiangsu Province 333 Project [grant number BRA2016145], the Natural Science Foundation of Jiangsu Province, China [grant number BK20160447], the Molecular Breeding Technology Construction of Public Service Platform, Yangzhou City [grant number YZ2017170], the Rice Industry Technology System of Yangzhou Comprehensive Experimental Station, Yangzhou, Jiangsu Province, China (CARS-01-60), and Natural Science Foundation of Yangzhou (YZ2017118)

Publisher's note Springer Nature remains neutral with regard to jurisdictional claims in published maps and institutional affiliations. 